\title{
Molecular and functional characterization of a novel sodium channel TipE-like auxiliary subunit from the American cockroach Periplaneta americana
}

Céline M. Bourdin ${ }^{\mathrm{a}}$, Nathalie C. Guérineau ${ }^{\mathrm{b}, \mathrm{c}}$, Laurence Murillo,d, Sophie Quinchard ${ }^{\mathrm{a}}, \mathrm{Ke}$ Dong $^{\mathrm{e}}$, Christian Legros ${ }^{\mathrm{c}, *}$

${ }^{a}$ Laboratoire Récepteurs et Canaux Ioniques Membranaires (RCIM) UPRES EA 2647 / USC INRA 1330, SFR QUASAV n 4207, Université d'Angers, UFR Sciences, 2 boulevard Lavoisier, F-49045 Angers CEDEX, France.

${ }^{\mathrm{b}}$ Laboratoire de Biologie Neurovasculaire et Mitochondriale Intégrée, CNRS UMR 6214, INSERM U1083, UFR de Sciences Médicales, Université d'Angers, rue Haute de Reculée, F49045 Angers CEDEX, France.

c present address : Institut de Génomique Fonctionnelle, UMR CNRS 5203, INSERM U661, Université de Montpellier, 141 rue de la Cardonille, F-34094 Montpellier CEDEX 5, France.

${ }^{\mathrm{d}}$ Laboratoire LIttoral ENvironnement et Sociétés (LIENSs), UMR 7266 CNRS Université de La Rochelle, Institut du Littoral et de l'Environnement, 2 rue Olympe de Gouges, F-17000 La Rochelle, France.

${ }^{\mathrm{e}}$ Department of Entomology, Genetics and Neuroscience Programs, Michigan State University, East Lansing, MI 48824, USA

${ }^{*}$ Corresponding author. Full postal address: Laboratoire de Biologie Neurovasculaire et Mitochondriale Intégrée, UMR CNRS 6214, INSERM 1083, UFR de Sciences Médicales, Université d'Angers, rue Haute de Reculée, F-49045 Angers CEDEX, France.

Phone: +33 (0) 2417358 34, Fax: +33 (0)2 41735895 .

E-mail address: christian.legros@univ-angers.fr 


\section{FOOTNOTES}

Abbreviations: voltage-gated sodium $\left(\mathrm{Na}_{\mathrm{v}}\right)$ channels; Basic Local Alignment Search Tool (BLAST); Rapid Amplification cDNA Ends (RACE). 


\section{Abstract}

In Drosophila melanogaster, the functions of voltage-gated sodium $\left(\mathrm{Na}_{\mathrm{v}}\right)$ channels are modulated by TipE and its orthologs. Here, we describe a novel TipE homolog of the American cockroach, Periplaneta americana, called PaTipE. Like DmTipE, PaTipE mRNAs are ubiquitously expressed. Surprisingly, PaTipE mRNA was undetectable in neurosecretory cells identified as dorsal unpaired median neurons. Phylogenetic analysis placed this new sequence in TipE clade, indicating an independent evolution from a common ancestor. Contrary to previous reports, our data indicate that the auxiliary subunits of insect $\mathrm{Na}_{\mathrm{v}}$ channels are very distant from the mammalian $\mathrm{BKCa}$ auxiliary subunits. To decipher the functional roles of PaTipE, we characterized the gating properties of $\mathrm{DmNa}_{\mathrm{v}} 1-1$ channels coexpressed with DmTipE or PaTipE, in Xenopus oocytes. Compared to DmTipE, PaTipE increased $\mathrm{Na}^{+}$currents by a 4.2-fold. The voltage-dependence of steady-state fast inactivation of $\mathrm{DmNa}_{\mathrm{v}} 1-1 /$ PaTipE channels was shifted by $5.8 \mathrm{mV}$ to more negative potentials than that of $\mathrm{DmNa}_{\mathrm{v}} 1-1 / \mathrm{DmTipE}$ channels. DmNa 1 1-1/PaTipE channels recovered 3.2-fold slower from the fast-inactivated state than $\mathrm{DmNa}_{\mathrm{v}} 1-1 / \mathrm{DmTipE}$ channels. In conclusion, this study supports that the insect $\mathrm{Na}_{\mathrm{v}}$ auxiliary subunits share functional features with their mammalian counterparts, although structurally and phylogenetically distant.

\section{Keywords}

Insect voltage-gated sodium channel; phylogenetic analysis; auxiliary subunit; Xenopus oocyte; two-electrode voltage-clamp technique. 


\section{Introduction}

Voltage-gated sodium $\left(\mathrm{Na}_{\mathrm{v}}\right)$ channels represent crucial plasma membrane components, that control depolarization-triggered fast $\mathrm{Na}^{+}$influx, allowing the generation and propagation of action potentials in excitable cells (Hille, 2001). $\mathrm{Na}_{\mathrm{v}}$ channels consist of a large pore-forming $\alpha$-subunit ( $260 \mathrm{kDa})$ and one or more smaller auxiliary proteins (Catterall et al., 2005). The $\mathrm{Na}_{\mathrm{v}}$ channel $\alpha$-subunit constitute molecular targets of numerous compounds such as, clinically used drugs for treatments of various diseases (e.g., epilepsy, chronic pain and cardiac arrhythmia), and also various animal and plant neurotoxins (Denac et al., 2000). Insect $\mathrm{Na}_{\mathrm{v}}$ channels are targeted by extensively used insecticides belonging to pyrethroid or pyrazoline families (Silver et al., 2010; Soderlund, 2008). However, these insecticides also impact the functions of mammalian $\mathrm{Na}_{\mathrm{v}}$ channel $\alpha$-subunits because of their high homology with insect counterparts.

In contrast, the $\mathrm{Na}_{\mathrm{v}}$ channels auxiliary subunits are more divergent in sequences and structure in both insects and mammals. In mammals, these auxiliary subunits, called $\beta$ subunits are glycoproteins containing a single transmembrane segment (Chahine and O'Leary, 2011). They modulate both trafficking and gating properties of $\mathrm{Na}_{\mathrm{v}}$ channels (Patino and Isom, 2010). The Drosophila melanogaster genome analysis has revealed the absence of gene encoding proteins homologous to the vertebrate $\mathrm{Na}_{\mathrm{v}}$ channel $\beta$-subunits (Littleton and Ganetzky, 2000). However, a family of five homologous genes encoding proteins (DmTipE, TEH1, TEH2, TEH3 and TEH4) have been previously reported in D. melanogaster with functions similar to those of mammalian $\beta$-subunits (Derst et al., 2006; Feng et al., 1995; Wang et al., 2015; Wang et al., 2013; Warmke et al., 1997). DmTipE is a glycosylated membrane protein $(\sim 65 \mathrm{kDa})$ that contains two membrane-spanning segments, encompassing a large extracellular loop and two intracellular extremities (Derst et al., 2006; Feng et al., 
1995). This topological organization is similar to that of $\beta$-subunits (Slo- $\beta$ ) of the mammalian big conductance calcium-activated potassium channel $\left(\mathrm{BK}_{\mathrm{Ca}}\right)$, suggesting a common ancestor (Derst et al., 2006).

The heterologous expression of insect $\mathrm{Na}_{\mathrm{v}}$ channel $\alpha$-subunits is a notoriously challenging task, limiting functional and pharmacological investigations. To date, the Xenopus oocyte expression system is the unique suitable system to heterologously express insect $\mathrm{Na}_{\mathrm{v}}$ channels. Only tiny currents are obtained when the $\mathrm{Na}_{\mathrm{v}}$ channel of $D$. melanogaster $\left(\mathrm{DmNa}_{\mathrm{v}} 1\right)$ is expressed alone in Xenopus oocyte (Feng et al., 1995; Warmke et al., 1997). By contrast, the co-expression of $\mathrm{DmNa}_{\mathrm{v}} 1$ with the auxiliary subunit DmTipE generates high $\mathrm{Na}^{+}$ current density that is associated with hastened inactivation kinetics (Derst et al., 2006; Feng et al., 1995; Warmke et al., 1997). The discovery of TipE in D. melanogaster has greatly improved the investigation of the molecular mechanisms of insecticides targeting $\mathrm{Na}_{\mathrm{v}}$ channels (Dong, 2007). DmTEH1 and DmTipE robustly boost $\mathrm{DmNa}_{\mathrm{v}} 1$ expression, resulting in 30-fold and 10-fold increases, respectively, in $\mathrm{Na}^{+}$current density. By comparison, DmTEH2 and DmTEH3 have weaker effects (5 to 8-fold increase in $\mathrm{Na}^{+}$currents), and DmTEH4 has no effect on $\mathrm{DmNa}_{\mathrm{v}} 1$ channel expression (Derst et al., 2006). In addition, when $\mathrm{DmNa}_{\mathrm{v}} 1$ is co-expressed with TipE or TEH1-4 subunits in Xenopus oocyte, elicited $\mathrm{Na}^{+}$ currents display distinct inactivation properties (Derst et al., 2006).

Two distinct types of $\mathrm{Na}_{\mathrm{v}}$ channels have been functionally characterized in the Dorsal Unpaired Median (DUM) neurons of the cockroach, Periplaneta americana, which is a commonly used neurophysiological model (Lavialle-Defaix et al., 2006; Lavialle-Defaix et al., 2010; Zhao et al., 2005). We hypothesized that these $\mathrm{Na}_{\mathrm{v}}$ channels differ by the composition of their auxiliary subunit. In this context, we cloned the $\mathrm{Na}_{\mathrm{v}}$ channel $\alpha$-subunit of P. americana, $\mathrm{PaNa}_{\mathrm{v}} 1$ (Moignot et al., 2009). Unfortunately, $\mathrm{PaNa}_{\mathrm{v}} 1$ is not expressible in Xenopus oocyte with DmTipE, DmTEH1 or PaTEH1 variants (Bourdin et al., 2013; Moignot 
et al., 2009). Then, $\mathrm{BgNa}_{\mathrm{v}} 1\left(\mathrm{Na}_{\mathrm{v}}\right.$ channel of Blattella germanica) and $\mathrm{DmNa}_{\mathrm{v}} 1$, sharing high amino acid sequence identities with $\mathrm{PaNa}_{\mathrm{v}} 1$ have been used in electrophysiological experiments in Xenopus oocytes. We have shown that the consequences of the interaction between $\alpha$-subunit and the auxiliary subunits of $P$. americana (PaTEH1) or D. melanosgaster (DmTEH1) are similar, demonstrating the functional conservation of these homologous auxiliary subunits (Bourdin et al., 2013). Like DmTEH1, PaTEH1 variants strongly increase $\mathrm{Na}_{\mathrm{v}}$ channel expression in Xenopus oocytes. PaTEH1 subunits also shift the voltagedependence of $\mathrm{Na}^{+}$currents of both activation and inactivation to more negative potentials, compared to those elicited by $\mathrm{BgNa}_{\mathrm{v}}$ alone (Bourdin et al., 2013). Thus, both activation and inactivation properties, as well as the expression level of $\mathrm{Na}_{\mathrm{v}}$ channels are modulated by coexpressed TipE-related proteins.

To extend the knowledge concerning insect $\mathrm{Na}_{\mathrm{v}}$ channel auxiliary subunits, we isolated cDNAs encoding the DmTipE homolog, PaTipE, in the nervous system of $P$. americana. We report here a phylogenetic analysis showing that this novel subunit is evolutionary closely related to others TipE family members. Reverse TranscriptionPolymerase Chain Reaction (RT-PCR) experiments revealed a conserved tissue distribution of PaTipE and DmTipE both within the central nervous system and in non-neuronal tissues. Electrophysiological experiments were next performed to characterize the influence of PaTipE on $\mathrm{DmNa}_{\mathrm{v}}$ channels expression and gating properties in Xenopus oocytes. 


\section{Material and methods}

\subsection{Anima/s}

American cockroaches ( $P$. americana) were reared in our laboratory under standard conditions $\left(29^{\circ} \mathrm{C}\right.$, photoperiod of 12 -h light/12-h dark). Xenopus laevis females used for oocytes preparations have been lab-bred. This study was carried out in strict accordance with the recommendations in the Guide for the Care and Use of Laboratory Animals of the European Community. The protocol was approved by the "Direction Départementale des services Vétérinaire du Maine et Loire" ( $\left.{ }^{\circ} \mathrm{B} 49071\right)$ and by the "Comité d'Ethique en Expérimentation Animale des Pays de la Loire" (NEEA.2012.68).

\subsection{Molecular biology}

Isolation of total RNA, reverse transcription (RT), PCR amplification and PCR product purification were performed as previously described (Chatel et al., 2013). The nucleotide sequences of primers used in our experiments are given in Table 1. For cloning a TipE-related partial cDNA of $P$. americana, a set of degenerated primers (TDP-S1 and TDP-R1) was designed from conserved amino acid sequences of TipE subunits of D. melanogaster, Musca domestica, Tribolium castaneum, Pediculus humanus corporis, Anopheles gambiae, Culex quinquefasciatus and Aedes aegypti. PCR experiments were carried out with EuroTaq polymerase (Eurobio, Courtaboeuf, France).

5'and 3' RACE experiments were performed as previously described (Moignot et al., 2009). Gene-specific primer sets used in RACE experiments were TP-R1, TP-R2, TP-S1 and TP-S2 (Table 1). The complete ORF of PaTipE cDNA was obtained by nested PCR using specific primers TP-S4 and TP-R4 followed by TP-S3 and TP-R3 (containing the restriction sites $\mathrm{Xmal}$ and $\mathrm{Xbal}$, respectively) and with a high fidelity hot start thermostable DNA polymerase (KOD HiFi DNA Polymerase; Novagen, Darmstadt, Germany). Because of the presence of a second ATG at 5' of PaTipE ORF, TP-S3 specific primer contained a silent 
single base change in a putative second start codon (ATG to ACG) at position 6 to avoid the translation of unwanted product. The amplicon of the second PCR was cloned into Xmal/Xbal digested pGEM-HEJUEL plasmid (kindly provided by Prof. Olaf Pongs, Institute for Neural Signal Transduction, Hamburg, Germany) and sequenced (GATC Biotech, Konstanz, Germany). Each clone was sequenced twice on both strands using universal sense and reverse primers. Sequences analyses were performed using BioEdit sequence analysis Software. The amino-acid sequences deduced from ORFs were identified using BLAST research in GenBank database (http://www.ncbi.nlm.nih.gov/blast/Blast.cgi) and by sequences alignment with DmTipE, performed using ClustalW method, as previously described (Moignot et al., 2009).

Semi-quantitative RT-PCR experiments were performed to address the tissue distribution pattern of PaTipE using TP-S3/R3 primer pair. Expression of PaTipE and PaTEH1 transcripts in DUM neurons was assessed by RT-PCR. The cytoplasm of 10 isolated DUM neurons were harvested via a patch-pipette mounted on a micromanipulator as described previously (Chatel et al., 2013). Total RNA was then purified using the Nucleospin ( R RNA XS (Macherey-Nagel, Hoerdt, Germany) and reverse-transcribed as described above. Nested amplification was performed with specific primer pairs encompassing ORF of PaTipE or PaTEH1 (Table 1). Amplicons were prepared as mentioned above and cloned into pGEMHE for sequence analysis. All RT-PCR experiments were carried out in triplicate.

\subsection{Phylogenetic analysis}

The accession numbers for cDNA sequences mentioned in this article are indicated in Table S1. The phylogenetic trees were constructed from TipE and TEHs auxiliary subunits. Fulllength ORFs were identified by Blast search in Genbank (Table S1). The alignment of amino acids deduced from DNA sequences was performed using ClustalW method (Thompson et al., 1994) implemented in MEGA5.0 (Tamura et al., 2011). The phylogenetic trees were then 
generated using Maximum likelihood method and confidence on node was assessed by bootstrapping 1000 times.

\subsection{Electrophysiological recording in Xenopus oocytes}

The cloning of $\mathrm{PaNa}_{\mathrm{v}} 1$ (GenBank ID: GQ132119) into pGEMHE has been already described by Moignot et al. (2009). DmNa $1-1$ and DmTipE subunits have been cloned into pGH19 as previously reported (Olson et al., 2008). pGEMHE and its derivative pGH19 contain both 5'and 3'-UTR from the Xenopus beta-globin gene, allowing high expression of foreign protein in Xenopus oocytes (Shih et al., 1998). All recombinant plasmids were linearized with Not/ (Invitrogen, Carlsbad, USA) or Spel (Promega, Madison, USA) and capped RNA were transcribed in vitro using the T7 mMESSAGEmMACHINE kit (Ambion, Austin, USA). The procedure for oocyte preparation was identical to that described previously (Bourdin et al., 2013). About 7 to $35 \mathrm{ng}$ of $\alpha$ and $\beta$ subunits RNA mixture were injected in oocytes (1:1 ratio). Injected oocytes were incubated in sterile medium composed of SOS supplemented with gentamycin $(50 \mu \mathrm{g} / \mathrm{ml})$, penicillin $(100 \mathrm{U} / \mathrm{ml})$, streptomycin $(100 \mu \mathrm{g} / \mathrm{ml})$ and sodium pyruvate $(2.5 \mathrm{mM})$ at $18^{\circ} \mathrm{C}$ for 3 to 10 days before recordings. Electrophysiological recording by twoelectrode voltage-clamp (TEVC) technique were performed as described in Bourdin et al. (2013). Briefly, oocytes were tested using $1 \mathrm{M}-\mathrm{KCl} / 2 \mathrm{M}-\mathrm{Kacetate}-$ filled borosilicate glass electrodes connected to a TEVC amplifier (TEV-200, Dagan Corporation, Minneapolis, Minnesota, USA). Digidata 1440A interface (Axon CNS Molecular Devices, California, USA) and pCLAMP 10 software (Axon CNS Molecular Devices) were used for acquisition and stimulation protocols. All experiments were performed at room temperature $\left(18-21^{\circ} \mathrm{C}\right)$ in standard oocyte saline composed of (in $\mathrm{mM}$ ): $\mathrm{NaCl} 96, \mathrm{KCl} 2, \mathrm{CaCl}_{2} 1.8, \mathrm{MgCl}_{2}$ 1, Hepes 5, $(\mathrm{pH} 7.5)$

For $\mathrm{Na}_{\mathrm{v}}$ channel activation, $\mathrm{Na}^{+}$current traces were generated by step depolarisations to test potentials from -70 to $40 \mathrm{mV}$ (10 mV increment, $20 \mathrm{~ms}$ duration) from a holding potential of - 
$100 \mathrm{mV}$, and $\mathrm{Na}_{\mathrm{v}}$ channel conductance $\left(\mathrm{G}_{\mathrm{Na}}\right)$ was calculated as already described (Barela et al., 2006). To determine the voltage-dependence of steady-state fast inactivation, $\mathrm{Na}^{+}$current traces were generated by a two-pulse protocol beginning with a prepulse step of depolarizing potential ranging from $-80 \mathrm{mV}$ to $40 \mathrm{mV}$ ( $5 \mathrm{mV}$ increment) for $200 \mathrm{~ms}$, followed by a second pulse to $-5 \mathrm{mV}$ for $12 \mathrm{~ms}$. Each set of double pulses was separated by $10 \mathrm{~s}$. For determination of the voltage-dependence of steady-state slow inactivation, a two-pulse protocol was used, starting with a prepulse of depolarizing potential ranging from $-90 \mathrm{mV}$ to $20 \mathrm{mV}(10 \mathrm{mV}$ increment) for $60 \mathrm{~s}$, followed by a $100 \mathrm{~ms}$ interval at the holding potential. A second pulse to $-5 \mathrm{mV}$ for $10 \mathrm{~ms}$ allowed measuring the available current. For both fast and slow inactivation, the peak current recorded during the second pulse was analysed as previously described (Lavialle-Defaix et al., 2006). For the recovery from inactivation, $\mathrm{Na}^{+}$currents were generated by a two-pulse protocol beginning with a first pulse to $-5 \mathrm{mV}$ for $50 \mathrm{~ms}$ followed by a second pulse to $-5 \mathrm{mV}$ for $14.5 \mathrm{~ms}$. The duration between the two pulses was increased by $1 \mathrm{~ms}$ every set of double pulses and each set was separated by $10 \mathrm{~s}$ (Lavialle-Defaix et al., 2006).

\subsection{Statistical analysis}

Statistical analysis of data was performed using GraphPad Prism software (version 5). All data are presented as mean \pm SEM. Significance tests between groups were performed using a variance analysis (one-way ANOVA) followed by a Tukey post hoc test for comparison of all groups, or the unpaired Student's $t$-test when appropriate. p values are specified. Statistical analysis probabilities are expressed as $*, p<0.05, * *, p<0.01$ and $* * *, p<0.001$.

\section{Results}

\subsection{Cloning and expression pattern of a novel TipE-like subunit of P. americana}


Since no sequence information was available in databases, for cloning TipE-related cDNA from $P$. americana, we designed two degenerated primers DTP-S1 and DTP-R1 (Table 1) targeting conserved regions which encompassed the extracellular loop of TipE-related proteins. A single 417-bp cDNA fragment was amplified and cloned from the nerve cord. It encoded a 139-amino acid polypeptide which was identified as the extracellular loop of a new TipE-like protein after blast analysis against non-redundant protein sequences (nr) database. Next, RACE and nested PCR experiments with gene-specific nested primers (Table 1) led us to identify a 214-bp 5'-untranslated region (5'UTR) and a 737-bp 3'-untranslated region (3'UTR), encompassing a 1065-pb ORF (Fig. S1 in the supporting information). A genespecific primer set (TP-S3 and TP-R3, Table 1) were designed to amplify the complete ORF, encoding a 354-amino-acid protein, called PaTipE (Fig. 1A) (GenBank ID: KC992733). The hydrophobicity profile of PaTipE revealed two hydrophobic domains (from position 20 to 41 and 226 to 249) that well aligned with the two putative transmembrane domains of DmTipE (Fig. 1B).

Semi-quantitative RT-PCR was performed in different tissues (head, thoracic ganglia, nerve cord, muscle, mushroom-shaped accessory gland and gut). Only one band of expected size (1065 bp) was observed in each tissue with a high intensity in heads, thoracic ganglia, nerve cords and muscle, and a lower intensity in mushroom-shaped accessory glands and gut (Fig. 1C). Surprisingly and contrasting with PaTEH1, PaTipE expression was undetectable in DUM neurons, even after two-round PCR, while PaTEH1 cDNA was amplified and its sequence was confirmed after cloning (Fig. 1D).

\subsection{Phylogenetic analysis}

The deduced amino acid sequence of PaTipE was aligned with those of TipE-like proteins from other insect species (Fig. S2 in the supporting information). PaTipE shares 
relative high sequence identities with TipE-like proteins of Apis florea (AfTipE, 64\%), Nasonia vitripennis (NvTipE, 63.6\%) and Bombus terrestris (BtTipE, 63.1\%), and lower sequence identities with TipE-like proteins of Diptera species such as D. melanogaster (42.1\%), Culex quinquefasciatus pipiens (41.9\%), Aedes aegypti (38.7\%) and Anopheles gambiae (34.8\%) (Table 2). Although the length of TipE-like protein varies among insect species (from 336 to 506 amino-acid residues), the amino acid sequences alignment clearly highlighted three distinct conserved regions. The first region comprises the N-terminal end, M1 segment and the first 60 amino acids of the extracellular loop. The second region includes the last 70-amino-acid residues of the extracellular loop N-terminal end and M2 segment followed by a cytosolic string of 10-amino-acid residues. The third region consists of the last 65-amino-acid residues of the C-terminal end. The two transmembrane regions are over $84 \%$ identical among all insect species, except for $A$. pisum (Table 2). The extracellular loop includes two highly conserved regions at their N- (52-53 amino acids) and C-termini (70-72 amino acids) separated by a variable region. The cytoplasmic N-termini are relatively short (19-22 amino acid residues) except for A. pisum (46 amino acids), while the C-termini display variable length among the insect species used in this study (102-167 amino acids). Interestingly, the C-termini end with a highly conserved string of 19 amino acids in all species, except in M. domestica and A. aegypti.

Aimed to establish the phylogenetic links between PaTipE and other insect auxiliary subunits, we first built a phylogram from insect TipE homologs (Fig. 2A). This analysis clearly indicated that PaTipE is phylogenetically related to eleven TipE homologs. The closest homologs of PaTipE are TcTipE from Coleoptera and PhumTipE from Phthiraptera, and afterwards NvTipE, BtTipE and AfTipE from Hymenoptera. Since a homology between TipE-related proteins of $D$. melanogaster and Slo- $\beta$ auxiliary subunits has been previously pointed out (Derst et al., 2006; Li et al., 2011), these sequences were implemented in a second 
analysis encompassing the four other subfamilies of insect $\mathrm{Na}_{\mathrm{v}}$ auxiliary subunits. The maximum likelihood method led to a single phylogram which was rooted at the branch between two major clusters (bootstrap values: 91 and 99, Fig. 2B). One cluster contains TipE, TEH1 and TEH2 homologs with high bootstrap values (99, 100, 99, respectively), strengthening the phylogenetic relationships between the three subfamilies. The second cluster assembles the two other subunits families, TEH3 and TEH4, with 90 and 95 bootstrap values. Altogether, Slo- $\beta 1$ to 4 sequences constitute a distant clade from the insect $\mathrm{Na}_{\mathrm{v}}$ auxiliary subunits, although both types of proteins share a similar membrane topology.

\subsection{Functional consequences of co-expression of DmNav1-1 with DmTipE or}

\section{PaTipE}

Because DmTipE is known to enhance the expression of $\mathrm{DmNa}_{\mathrm{v}} 1$ or $\mathrm{BgNa}_{\mathrm{v}} 1$ channels in Xenopus oocytes, we assumed that it could also increase $\mathrm{Na}^{+}$current when co-expressed with $\mathrm{PaNa}_{\mathrm{v}} 1$. Then, we measured $\mathrm{Na}^{+}$current elicited by $\mathrm{PaNa}_{\mathrm{v}} 1$ channels co-expressed with DmTipE or PaTipE. Unfortunately, the oocytes injected with $\mathrm{PaNa}_{\mathrm{v}} 1 \mathrm{mRNA}$ (35 ng or up to $50 \mathrm{ng}$ and incubated up to 15 days) did not generate any detectable currents with or without DmTipE or PaTipE (Fig. 3A). By contrast, we detected small $\mathrm{Na}^{+}$currents $(0.25 \mu \mathrm{A}, 10$-days incubation, $35 \mathrm{ng}$ of mRNA) from oocytes injected with mRNA of $\mathrm{DmNa}_{\mathrm{v}} 1-1$ alone, and much larger $\mathrm{Na}^{+}$currents from oocytes co-injected with mRNA of DmTipE or PaTipE (Fig. 3A). Fig. 3B summarizes the quantitative analysis of $\mathrm{Na}^{+}$current density measured from oocytes injected with mRNA of $\mathrm{DmNa}_{\mathrm{v}} 1-1$ alone or in combination with mRNA of DmTipE, PaTipE or DmTEH1. When compared to the expression of $\mathrm{DmNa}_{\mathrm{v}} 1-1$ alone, all tested auxiliary subunits enhanced $\mathrm{Na}^{+}$channel current density, although with different efficiencies. The highest $\mathrm{Na}^{+}$current density was obtained with PaTipE $(0.75 \pm 0.09 \mu \mathrm{A} / \mathrm{nF} / \mathrm{ng}$ of injected RNA). Lower $\mathrm{Na}^{+}$current densities were recorded with DmTEH1 and DmTipE $(0.47 \pm 0.06$ and $0.18 \pm 0.03 \mu \mathrm{A} / \mathrm{nF} / \mathrm{ng}$ of injected RNA, respectively, Fig. 3B). In the presence of PaTipE, 
the $\mathrm{Na}^{+}$current density was increased by 22.8 fold compared to $\mathrm{DmNa}_{\mathrm{v}} 1-1$ alone. Coexpression of $\mathrm{DmNa}_{\mathrm{v}} 1-1$ with DmTipE or DmTEH1 induced an increase of $\mathrm{Na}^{+}$current densities by $5.4 \pm 0.9(\mathrm{p}>0.05)$ and $14.1 \pm 1.7$ fold $(\mathrm{p}<0.001)$, respectively. The finding that PaTipE more efficiently facilitates $\mathrm{DmNa}_{\mathrm{v}} 1$-1-elicited $\mathrm{Na}^{+}$currents than DmTipE (4.2-fold) suggests that PaTipE subunit may display more robust chaperone properties.

Next, we examined the biophysiological properties of $\mathrm{DmNa}_{\mathrm{v}} 1-1 / \mathrm{DmTipE}$ and $\mathrm{DmNa}_{\mathrm{v}} 1-1 /$ PaTipE channels in Xenopus oocytes (Fig. 4). We observed no significant difference in the voltage dependence of activation between $\mathrm{DmNa}_{\mathrm{v}} 1-1 / \mathrm{DmTipE}$ and $\mathrm{DmNa}_{\mathrm{v}} 1$ 1/PaTipE channels (Fig. 4A, Table 3). Compared with DmTipE, PaTipE shifted the voltage dependence of fast inactivation to more negative potentials (by $5.8 \mathrm{mV}, \mathrm{p}<0.05$, Fig. 4B, Table 3), but did not modify the voltage dependence of slow inactivation (Fig. 4C, Table 3). In addition, the recovery from steady-state fast inactivation was 3.2-fold slower for $\mathrm{DmNa}_{\mathrm{v}} 1$ 1/PaTipE channels $(\tau=3.8 \pm 0.3 \mathrm{~ms})$ than $\operatorname{DmNa}_{\mathrm{v}} 1-1 / \mathrm{DmTipE}$ channels $(\tau=1.2 \pm 0.1 \mathrm{~ms}$, Fig. 4D, Table 3).

\section{Discussion}

TipE subunit and its homologs are relevant components of insect $\mathrm{Na}_{\mathrm{v}}$ channels, enabling changes in channel expression, gating and pharmacological properties (Bourdin et al., 2013; Derst et al., 2006; Feng et al., 1995; Wang et al., 2015; Wang et al., 2013; Warmke et al., 1997). Here, we cloned a novel auxiliary subunit, PaTipE, from the American cockroach, P. americana, which is phylogenetically affiliated to DmTipE. Both subunits share similar chaperone properties when co-expressed with $\mathrm{DmNa}_{\mathrm{v}} 1-1$ in Xenopus oocytes. Like DmTipE, PaTipE robustly increases $\mathrm{Na}^{+}$current density of $\mathrm{DmNa}_{\mathrm{v}} 1-1$ channels. In addition, the biophysical properties of $\mathrm{DmNa}_{\mathrm{v}} 1-1 / \mathrm{DmTipE}$ and $\mathrm{DmNa}_{\mathrm{v}} 1-1 /$ PaTipE channels are 
similar. However, their fast inactivation properties slightly differ in voltage-dependency and recovery from steady-state inactivation.

We showed that PaTipE mRNA is broadly expressed in $P$. americana in both neuronal and non-neuronal tissues, like DmTipE in D. melanogaster (Derst et al., 2006). The highest expression level was detected whithin the central nervous system, matching thus the tissue distribution of PaNav1 (Moignot et al., 2009). We therefore propose that TipE-like gene exhibit ubiquitous expression in other insect species and display similar functions. Likewise, DmTEH1 subunit and its homolog in P. americana, PaTEH1, share similar neuronal expression patterns (Bourdin et al., 2013). Interestingly, PaTipE, PaTEH2 and PaTEH3 (unpublished data) are not expressed in DUM neurons. Since only PaTEH1 is expressed in DUM neurons in which two $\mathrm{Na}_{\mathrm{v}}$ channels have been previously characterized by patch-clamp technique (Lavialle-Defaix et al., 2006; Lavialle-Defaix et al., 2010; Zhao et al., 2005), we suggest that this functional diversity is endorsed by the presence of two $\alpha$-subunits, resulting from splicing or RNA editing events associated with PaTEH1. Indeed, extensive alternative splicing and RNA editions are believed to be responsible of a fine tuning of $\mathrm{Na}_{\mathrm{v}}$ channels gating properties in B. germanica and D. melanogaster(Olson et al., 2008; Song et al., 2004).

Although TipE-like proteins display various lengths among insect species, the amino acid sequence alignment clearly highlights three highly conserved regions and two variable segments. This prompts us to hypothesize that these conserved regions are important for $\mathrm{Na}_{\mathrm{v}}$ channel modulation and/or function. Our phylogenetic analysis points out that TipE homologs cluster within a distinct sub-clade from TEH1 and $\mathrm{TEH} 2$, indicating that TipE has independently evolved from a common ancestor. Phylogram indicates that the closest members of the TipE sub-clade are those from Coleoptera (T. castaneum) and Phthiraptera $(P$. humanus corporis). Additionally, we report that mammalian Slo- $\beta 1$ to $\beta 4$ subunits are phylogenetically distant from insect $\mathrm{Na}_{\mathrm{v}}$ auxiliary subunits. This raises the question whether 
these two types of auxiliary subunits have evolved from a common ancestor or result of a convergent evolution. However, since both TipE-like proteins and Slo- $\beta$ auxiliary subunits share similar membrane topology and $1 / 2$ cysteine patterns, we suggest that they have evolved from an old common molecular ancestor. This is in agreement with previous studies reporting sequence similarity between TipE-related proteins of $D$. melanogaster and Slo- $\beta$ auxiliary subunits (Derst et al., 2006; Li et al., 2011).

DmTipE is the first insect auxiliary subunit described for its ability to enhance $\mathrm{Na}^{+}$ currents when co-expressed with $\mathrm{Na}_{\mathrm{v}}$ - -subunit in Xenopus oocytes, indicating that this protein display a chaperone-like or stabilizing effects on channel expression (Derst et al., 2006). Surprisingly, $\mathrm{Na}^{+}$current densities are much higher when PaTipE, rather than DmTipE, is co-expressed with $\mathrm{DmNa}_{\mathrm{v}}$. Such a species-dependent difference has also been observed with TEH1-like subunit (Bourdin et al., 2013). Further structure-function relationship studies are needed to decipher the molecular basis of this difference. Our results show that, the voltage-dependence of activation of $\mathrm{Na}^{+}$currents generated by $\mathrm{DmNa}_{\mathrm{v}} 1-$ 1/DmTipE or $\mathrm{DmNa}_{\mathrm{v}} 1-1 / \mathrm{PaTipE}$ channels is indistinguishable. These findings, in addition to other studies (Silver et al., 2010; Warmke et al., 1997) strongly suggest that activation properties of $\mathrm{Na}_{\mathrm{v}}$ channels do not depend on the auxiliary TipE subunit. Similar observations have been reported for the $\mathrm{Na}_{\mathrm{v}}$ channels of Musca domestica, Vssc1/Vssc $\beta$ (Lee et al., 2000). With respect to inactivation properties, only the fast inactivation differs significantly between $\mathrm{DmNa}_{\mathrm{v}} 1-1 /$ DmTipE and $\mathrm{DmNa}_{\mathrm{v}} 1-1 /$ PaTipE channels. When co-expressed with PaTipE, $\mathrm{DmNa}_{\mathrm{v}} 1-1$ displayed a more negative voltage-dependence of fast inactivation $(-5.8 \mathrm{mV})$ and recovered 3.2-fold slower from steady-state fast inactivation compared to the $\mathrm{DmNa}_{\mathrm{v}} 1$ 1/DmTipE combination. This could reflect the differences in amino acid sequences of both auxiliary subunits. We also suggest that TipE subunits are involved in the modulation of fast inactivation properties of $\mathrm{Na}_{\mathrm{v}}$ channels. Recently, Wang and colleagues reported that DmTipE 
impacts the voltage dependence of fast inactivation of only one $\mathrm{DmNa}_{\mathrm{v}}$ variant out of the three variants examined (Wang et al., 2013), reinforcing thus the idea that TipE-like proteins are fast inactivation regulators, but their effects on gating may depend on $\mathrm{Na}^{+}$channel variants.

In conclusion, the insect $\mathrm{Na}_{\mathrm{v}}$ auxiliary subunits PaTipE and DmTipE display similar functional characteristics (chaperon-like effects and inactivation properties) and an ubiquitous tissue distribution. The activation properties of $\mathrm{Na}_{\mathrm{v}}$ channels co-expressed with both auxiliary subunits are similar, but their fast inactivation properties slightly differ by their voltagedependency and stability. These differences point the functional importance of the speciesspecific interaction between auxiliary subunits and native $\mathrm{Na}_{\mathrm{v}}$ channels. The TipE homologous genes constitute a phylogenetical distinct sub-clade originated from a common ancestry, which is clearly distant from the unique structurally related proteins found in mammals, that is the Slo- $\beta$ auxiliary subunit family. In regard to the absence of mammalian counterpart of insect auxiliary subunits, and their low amino acid sequence identity, it will be of a great interest to investigate the structure-function relationship of TipE homologs with the idea to develop insect species-specific insecticides.

\section{Acknowledgements}

C.M. Bourdin was supported by a doctoral fellowship of the French National Institute for Agricultural Research (INRA) and Région Pays de la Loire.

\section{List of supplementary on-line material}

- Table S1

- Figure S1

- Figure S2 


\section{References}

Barela, A.J., Waddy, S.P., Lickfett, J.G., Hunter, J., Anido, A., Helmers, S.L., Goldin, A.L., Escayg, A., 2006. An epilepsy mutation in the sodium channel SCN1A that decreases channel excitability. J Neurosci 26, 2714-2723.

Bourdin, C.M., Moignot, B., Wang, L., Murillo, L., Juchaux, M., Quinchard, S., Lapied, B., Guérineau, N.C., Dong, K., Legros, C., 2013. Intron retention in mRNA encoding ancillary subunit of insect voltage-gated sodium channel modulates channel expression, gating regulation and drug sensitivity. PLoS One 8, e67290.

Catterall, W.A., Goldin, A.L., Waxman, S.G., 2005. International Union of Pharmacology. XLVII. Nomenclature and structure-function relationships of voltage-gated sodium channels. Pharmacological reviews 57, 397-409.

Chahine, M., O'Leary, M.E., 2011. Regulatory Role of Voltage-Gated Na Channel beta Subunits in Sensory Neurons. Frontiers in pharmacology 2, 70.

Chatel, A., Murillo, L., Bourdin, C.M., Quinchard, S., Picard, D., Legros, C., 2013. Characterization of tyramine beta-hydroxylase, an enzyme upregulated by stress in Periplaneta americana. J Mol Endocrinol 50, 91-102.

Denac, H., Mevissen, M., Scholtysik, G., 2000. Structure, function and pharmacology of voltage-gated sodium channels. Naunyn-Schmiedeberg's archives of pharmacology 362, 453-479.

Derst, C., Walther, C., Veh, R.W., Wicher, D., Heinemann, S.H., 2006. Four novel sequences in Drosophila melanogaster homologous to the auxiliary Para sodium channel subunit TipE. Biochem Biophys Res Commun 339, 939-948.

Dong, K., 2007. Insect sodium channels and insecticide resistance. Invert Neurosci 7, 17-30.

Feng, G., Deak, P., Chopra, M., Hall, L.M., 1995. Cloning and functional analysis of TipE, a novel membrane protein that enhances Drosophila para sodium channel function. Cell 82, 1001-1011.

Hille, B., 2001. Ion Channels of Excitable Membranes. . Sinauer Associates, Inc., Sunderland, MA.

Lavialle-Defaix, C., Gautier, H., Defaix, A., Lapied, B., Grolleau, F., 2006. Differential regulation of two distinct voltage-dependent sodium currents by group III metabotropic glutamate receptor activation in insect pacemaker neurons. J Neurophysiol 96, 2437-2450.

Lavialle-Defaix, C., Moignot, B., Legros, C., Lapied, B., 2010. How does calcium-dependent intracellular regulation of voltage-dependent sodium current increase the sensitivity to the oxadiazine insecticide indoxacarb metabolite decarbomethoxylated JW062 (DCJW) in insect pacemaker neurons? The Journal of pharmacology and experimental therapeutics 333, 264-272.

Lee, S.H., Smith, T.J., Ingles, P.J., Soderlund, D.M., 2000. Cloning and functional characterization of a putative sodium channel auxiliary subunit gene from the house fly (Musca domestica). Insect Biochem Mol Biol 30, 479-487.

Li, J., Waterhouse, R.M., Zdobnov, E.M., 2011. A remarkably stable TipE gene cluster: evolution of insect Para sodium channel auxiliary subunits. BMC evolutionary biology 11, 337.

Littleton, J.T., Ganetzky, B., 2000. Ion channels and synaptic organization: analysis of the Drosophila genome. Neuron 26, 35-43.

Moignot, B., Lemaire, C., Quinchard, S., Lapied, B., Legros, C., 2009. The discovery of a novel sodium channel in the cockroach Periplaneta americana: evidence for an early duplication of the para-like gene. Insect Biochem Mol Biol 39, 814-823.

Olson, R.O., Liu, Z., Nomura, Y., Song, W., Dong, K., 2008. Molecular and functional characterization of voltage-gated sodium channel variants from Drosophila melanogaster. Insect Biochem Mol Biol 38, 604-610.

Patino, G.A., Isom, L.L., 2010. Electrophysiology and beyond: multiple roles of $\mathrm{Na}+$ channel beta subunits in development and disease. Neurosci Lett 486, 53-59.

Shih, T.M., Smith, R.D., Toro, L., Goldin, A.L., 1998. High-level expression and detection of ion channels in Xenopus oocytes. Methods Enzymol 293, 529-556. 
Silver, K.S., Song, W., Nomura, Y., Salgado, V.L., Dong, K., 2010. Mechanism of action of sodium channel blocker insecticides (SCBIs) on insect sodium channels. Pestic Biochem Physiol 97, 87-92.

Soderlund, D.M., 2008. Pyrethroids, knockdown resistance and sodium channels. Pest management science 64, 610-616.

Song, W., Liu, Z., Tan, J., Nomura, Y., Dong, K., 2004. RNA editing generates tissue-specific sodium channels with distinct gating properties. J Biol Chem 279, 32554-32561.

Tamura, K., Peterson, D., Peterson, N., Stecher, G., Nei, M., Kumar, S., 2011. MEGA5: molecular evolutionary genetics analysis using maximum likelihood, evolutionary distance, and maximum parsimony methods. Mol Biol Evol 28, 2731-2739.

Thompson, J.D., Higgins, D.G., Gibson, T.J., 1994. CLUSTAL W: improving the sensitivity of progressive multiple sequence alignment through sequence weighting, position-specific gap penalties and weight matrix choice. Nucleic Acids Res 22, 4673-4680.

Wang, L., Du, Y., Nomura, Y., Dong, K., 2015. Distinct modulating effects of TipE-homologs 2-4 on Drosophila sodium channel splice variants. Insect Biochem Mol Biol 60, 24-32.

Wang, L., Nomura, Y., Du, Y., Dong, K., 2013. Differential effects of TipE and a TipE-homologous protein on modulation of gating properties of sodium channels from Drosophila melanogaster. PLoS One 8, e67551.

Warmke, J.W., Reenan, R.A., Wang, P., Qian, S., Arena, J.P., Wang, J., Wunderler, D., Liu, K., Kaczorowski, G.J., Van der Ploeg, L.H., Ganetzky, B., Cohen, C.J., 1997. Functional expression of Drosophila para sodium channels. Modulation by the membrane protein TipE and toxin pharmacology. J Gen Physiol 110, 119-133.

Zhao, X., Ikeda, T., Salgado, V.L., Yeh, J.Z., Narahashi, T., 2005. Block of two subtypes of sodium channels in cockroach neurons by indoxacarb insecticides. Neurotoxicology 26, 455-465. 


\section{Figure legends}

Figure 1. Cloning and molecular characterization of PaTipE subunit of $P$. americana. A. Alignment of deduced amino acid sequences of the PaTipE and DmTipE proteins. Transmembrane segments (M1 and M2) are indicated with bold line above the sequences. Conserved N-glycosylation sites are indicated by asterisk $\left(^{*}\right)$ (www.cbs.dtu.dk). Gaps are indicated by dashes. B. Hydrophobicity profile and deduced topological organization of PaTipE (left) and DmTipE (right). Hydrophobicity analysis was performed using the algorithm of Kyte and Doolittle (1982). The amino acid residue position is plotted along the $\mathrm{x}$-axis and the calculated mean hydrophobicity is plotted along the $\mathrm{y}$-axis. Regions above the dashed line are hydrophobic. The two putative membrane-spanning segments of both PaTipE and DmTipE are indicated (M1 and M2). C. Semi-quantitative RT-PCR analysis of PaTipE expression in various $P$. americana tissues. RT-PCR was performed using $5 \mu \mathrm{g}$ of mRNA extracted from heads, thoracic ganglia, nerve cords, muscles, gut and mushroom-shaped accessory glands (Msag). D. Expression analysis of PaTipE and PaTEH1 mRNA in DUM neurons by RT-PCR. No transcript was found for PaTipE. Conversely, PaTEH1 mRNA was detected as a single band and its sequence was identical to PaTEH1C variant (Genbank ID: KC247670). Actin (Genbank ID : AY116670) was used as an internal quantitative control.

Figure 2. Phylogenetic analyses of TipE-like protein family. A. Sequences of TipE and TipErelated (TEH) proteins of D. melanogaster, Musca domestica, Anopheles gambiae, Aedes aegypti, Culex quinquefasciatus pipiens, Tribolium castaneum, Pediculus humanus corporis, Apis florea, Bombus terrestris, Acyrthosiphon pisum and P. americana were aligned using ClustalW. B. Phylogenetic tree of TipE-like protein family and human Slo- $\beta$ subunits proteins. All sequences were aligned using ClustalW. Human $\beta$-slo subunits accession numbers are: hslobeta1, NM_004137; hslobeta2, NM_005832; hslobeta3, NM_171828; hslobeta4, NM_014505. Bootstrap values are indicated and the scale bar represents 
substitutions per site. The accession numbers of the sequences included in the tree are listed in the Table S1.

Figure 3. Distinct effect of the auxiliary subunit DmTipE, PaTipE or DmTEH1 on $\mathrm{Na}^{+}$ currents evoked by PaNav1 and $\mathrm{DmNa}_{\mathrm{v}} 1-1$ channel activation. A. Chart recordings of of $\mathrm{Na}^{+}$ currents measured at test potentials of $-70 \mathrm{mV}$ to $40 \mathrm{mV}$ from a holding potential of $-100 \mathrm{mV}$, in response to the expression of $\mathrm{PaNa}_{\mathrm{v}} 1$ alone, $\mathrm{PaNa}_{\mathrm{v}} 1 /$ DmTipE, $\mathrm{PaNa}_{\mathrm{v}} 1 / \mathrm{PaTipE}, \mathrm{DmNa}_{\mathrm{v}} 1-1$ alone, $\mathrm{DmNa}_{\mathrm{v}} 1-1 / \mathrm{DmTipE}_{\mathrm{N}} \mathrm{DmNa}_{\mathrm{v}} 1-1 /$ PaTipE in Xenopus oocytes injected with about 11 ng, $7.4 \mathrm{ng}, 5 \mathrm{ng}, 35 \mathrm{ng}, 7 \mathrm{ng}$ and $3 \mathrm{ng}$ of RNA (1:1 ratio), respectively. B. $\mathrm{Na}^{+}$current density ( $\mu \mathrm{A}$ per ng of injected RNA) after 3-day incubation, except for $\mathrm{DmNa}_{\mathrm{v}} 1-1$ (10 days after injection). The number of recorded oocytes is indicated in the bar histogram.

Figure 4. Biophysical properties of $\mathrm{Na}^{+}$currents elicited by co-expression of $\mathrm{DmNa}_{\mathrm{v}} 1-$ 1/DmTipE and $\mathrm{DmNa}_{\mathrm{v}} 1-1 / \mathrm{PaTipE}$ channels. A. Voltage-dependences of activation of $\operatorname{DmNa}_{v} 1-1 / \operatorname{DmTipE}\left(n=6\right.$, filled circles) and $\operatorname{DmNa}_{v} 1-1 / \operatorname{PaTipE}(n=7$, open squares $) . \mathrm{G}$ represents the conductance. B. Voltage dependence of fast steady-state inactivation of $\operatorname{DmNa}_{v} 1-1 / \operatorname{DmTipE}(n=7)$ and $\operatorname{DmNa}_{v} 1-1 /$ PaTipE $(n=7)$ C. Voltage-dependence of slow steady-state inactivation of $\operatorname{DmNa}_{\mathrm{v}} 1-1 / \operatorname{DmTipE}(n=5)$ and $\operatorname{DmNa}_{\mathrm{v}} 1-1 / \operatorname{PaTipE}(\mathrm{n}=4)$. D. Recovery from fast inactivation of $\operatorname{DmNa}_{\mathrm{v}} 1-1 / \operatorname{DmTipE}(\mathrm{n}=6)$ and $\mathrm{DmNa}_{\mathrm{v}} 1-1 / \mathrm{PaTipE}$ $(\mathrm{n}=7) . \mathrm{Na}^{+}$current amplitudes (I) were measured using the pulse protocols described in the Materials and Methods section and were normalized to the largest current amplitude (Imax). Standard protocols are shown in inset. Values are mean \pm SEM.

Figure S1. Composite nucleotide sequence of PaTipE and its complete deduced amino acid sequence. The open reading frame begins at nucleotide 215 (sequence ATG: M) and closes at 
nucleotide 1279 (TGA). A putative polyadenylation signal is underlined. The numbers on the right indicate the position of the nucleotides.

Figure S2. Amino acid sequences alignment of TipE homologs. Identical and similar amino acids are highlighted in dark grey and grey, respectively. Putative transmembrane regions are boxed. Conserved half-cystines are indicated by an arrow. The accession numbers of the sequences included in the alignment are listed in the Table S1. 
Table 1

Sequences of the oligonucleotides used in PCR and their corresponding region

\begin{tabular}{|c|c|c|}
\hline Primers name & Nucleotide sequence & Domain \\
\hline \multicolumn{3}{|c|}{ 1-Degenerated primer used to amplify PaTipE } \\
\hline TDP-S1 & 5'-CCBGCNTTCACSACDATYTTCATG-3' & MS1-MS2 \\
\hline TDP-R1 & 5'-RTAVCCRCADCCYTTBACRTTBGG-3' & MS1-MS2 \\
\hline \multicolumn{3}{|c|}{ 2-Specific primers used in RACE } \\
\hline TP-R1 & 5'-CCACTCCGAGTCGTTCCCCAT-3' & 5'UTR partial ORF PaTipE(PCR\#1) \\
\hline TP-R2 & 5'-TCTCTTGGACCGCAGATATTCGTG-3' & 5'UTR partial ORF PaTipE(PCR\#2) \\
\hline TP-S1 & 5'-GTGGCGGAGCTCCACGAATATCTGC-3' & 3'UTR partial ORF PaTipE(PCR\#1) \\
\hline TP-S2 & 5'-GGACTCATGGGGAACGACTCGGA -3' & 3'UTR partial ORF PaTipE(PCR\#2) \\
\hline \multicolumn{3}{|c|}{ 3-Primers used to amplify the full-length ORF } \\
\hline TP-S3 & 5'-AAACCCGGGCCAACCATGGACGAGCCGGAGATTGAGC-3' & Full-length ORF PaTipE \\
\hline TP-S4 & 5'-GGGTGCATTCAAAGCACGATGACT-3' & Complete cDNA PaTipE \\
\hline TP-R3 & 5'-CAAAA TCTAGATCAGACTTCCGCTATCGGCCCAG-3' & Full-length ORF PaTipE \\
\hline TP-R4 & 5'-CCACACAAGGTTAAAGCCTGTGGC-3' & Complete cDNA PaTipE \\
\hline TP-S5 & 5'- AAAAGTTGTCAGCTGTTCGGCG-3' & Complete cDNA PaTEH1 \\
\hline TP-S6 & 5'-CAGAT CCCGGGATGAGGAGCAGCAGCTCGGAG-3' & Full-length ORF PaTEH1 \\
\hline TP-R5 & 5'-(T)23CAAAATATAGGCCATGTATTTCTACC-3' & Complete cDNA PaTEH1 \\
\hline TP-R6 & 5'-CTGAT TCTAGACCGAATCATGTTCTATCTTCT-3' & Full-length OFR PaTEH1 \\
\hline
\end{tabular}

Designation of oligonucleotide mixtures: $\mathrm{R}=\mathrm{G}+\mathrm{A} ; \mathrm{S}=\mathrm{G}+\mathrm{C} ; \mathrm{Y}=\mathrm{C}+\mathrm{T} ; \mathrm{M}=\mathrm{A}+\mathrm{C} ; \mathrm{B}=\mathrm{G}+\mathrm{T}+\mathrm{C} ; \mathrm{D}=\mathrm{G}+\mathrm{A}+\mathrm{T} ; \mathrm{V}=\mathrm{G}+\mathrm{A}+\mathrm{C}$

$\mathrm{N}=\mathrm{G}+\mathrm{A}+\mathrm{T}+\mathrm{C}$.

UTR, untranslated region; ORF, open-reading frame

Restriction enzyme recognition sequences are underlined and correspond to XmaI site (CCCGGG) and XbaI site (TCTAGA). 
Table 2

Amino acid sequence homology between PaTipE and TipE-like protein of others species.

\begin{tabular}{lcccccc}
\hline \multirow{2}{*}{ Species } & N-terminal & TM1 & Extracellular loop & TM2 & C-terminal & Total \\
\cline { 2 - 7 } & \% Amino acid identity with PaTipE & & & \\
\cline { 2 - 7 } D. melanogaster & 52.3 & 88 & 41.9 & 90.9 & 31.6 & 42.1 \\
M. domestica & 57.1 & 84 & 37.6 & 86.3 & 20.7 & 36 \\
A. aegyptii & 61.9 & 84 & 34.9 & 95.4 & 24.6 & 38.7 \\
A. gambiae & 61.9 & 88 & 32.1 & 95.4 & 33.1 & 34.8 \\
C. quinquefasciatus pipiens & 66.6 & 92 & 34.5 & 86.3 & 34.7 & 41.9 \\
N. vitripennis & 59 & 92 & 60.4 & 95.4 & 53 & 63.6 \\
A. florea & 66.6 & 88 & 57.9 & 95.4 & 59.2 & 64 \\
B. terrestris & 66.6 & 88 & 58.5 & 95.4 & 55.7 & 63.1 \\
A. pisum & 13 & 72 & 31.3 & 54.5 & 17.9 & 25.4 \\
P. humanus corporis & 52.1 & 88 & 45.2 & 86.3 & 46.5 & 53.9 \\
T. castaneum & 76.1 & 92 & 46.5 & 86.3 & 53.2 & 54.1 \\
\hline
\end{tabular}




\section{Table 3}

Parameters of voltage-dependence of activation, fast and slow steady-state inactivation, and recovery from fast inactivation for $\mathrm{DmNa}_{\mathrm{v}} 1-1$ co-expressed with DmTipE or PaTipE in Xenopus oocytes.

\begin{tabular}{llllllll}
\hline & Activation & & \multicolumn{2}{c}{ Fast inactivation } & \multicolumn{2}{c}{ Slow inactivation } & \multicolumn{2}{c}{ Recovery } \\
\hline & $\mathrm{V} 1 / 2(\mathrm{mV})$ & $\mathrm{k}$ & $\mathrm{V} 1 / 2(\mathrm{mV})$ & $\mathrm{k}$ & $\mathrm{V} 1 / 2(\mathrm{mV})$ & $\mathrm{k}$ & $\tau(\mathrm{ms})$ \\
\hline DmTipE & $-20.2 \pm 1.4(5)$ & $10.6 \pm 1.3$ & $-47.3 \pm 0.5(7)$ & $7.1 \pm 0.4$ & $-51.1 \pm 1.0(4)$ & $6.8 \pm 0.9$ & $1.2 \pm 0.1(6)$ \\
PaTipE & $-15.7 \pm 2.0(7)$ & $14.4 \pm 2.1$ & $-53.1 \pm 0.5(5)^{\mathrm{a}}$ & $6.5 \pm 0.4$ & $-54.6 \pm 0.9(7)$ & $8.9 \pm 0.8$ & $3.8 \pm 0.3(5)^{\mathrm{b}}$ \\
\hline
\end{tabular}

Values are mean \pm SEM derived from the number of individual experiments, each performed with a different oocyte. The number of recorded oocytes is indicated in parenthesis

${ }^{a}$ significant difference $\mathrm{p}<0.05$ compared to DmTipE

${ }^{\mathrm{b}}$ Significant difference $\mathrm{p}<0.001$ compared to DmTipE 
PaTIPE MDEPEIEQTLAQKLLFYTTAFFVLLGTFSLFAFLFLVPFVIDPAFTTIFMQFDPVAALCQ 60

DmTiPE MGDEQDKRTGKEKLLFYTTAFFILLGTFSLFAFLFLVPFVIEPAFTTIFMQFEEVPALCE 60

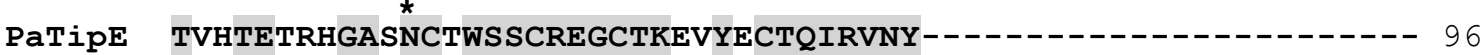

DMTIPE TYDTEIYYGAKNCSWSSCREGCTKDIYTCTQIRVNYRLNLYNFTDEFNFTEYHINLKEAE 120

PaTIPE KILPPVKQEEGEE-------GNAGEDEGTDEDIVAELHEYLRSKRAIRDYDYSSEDEE 147

DMTiPE RILPPVKRTDRYERALRSDYEYDNLGGGTGLDIDLGAGRMEQLNFGDADGSNGYLIEDSE 180

PaTipE --------IALQEPEP-------TGLMGNDSEWYYTQAKLFPNVKGCGYPPMLNCSI 189

DmTiPE DTRGLSASGTLISDERRPFDEISELNEGLMGNRSMYYYVGARLFPNVKGCGYPPMLNCTI 240

M2

PaTiPE FVKMYSEPGTNFSCYYSKVDPGLVMSELDMWQVYMNLVYAMAIPIPSFIISVIYLTFAYF 249

DmTiPE WLKRYTKIGMKFPCYYSKVDPSLVISDLDYWQNTLNLVYSMAIPIPSFIISVIYLTYAYF 300

PaTipE KIYNDDDEEALVN-------------------AAG------ETVEGDAAENGVT 278

DmTIPE KIYNEDEETAPLDKNAEDMDIDDIDAVDDSDGAVLADNVAGSQI INMDSTTNDSCLEGVL 360

PaTipE P------VPPLTSG--AITPGSEIFR---------EDLASFGHQLKVAMADEMSRESVA 320

DmTIPE PNGGPGMTASISQGGSVTTPGPYIAQSPAGSQMTPNSEINSFGHQLKVQMADELSRDSLE 420

PaTiPE DAVPVIANSNSIPGNLSKTMTTSISTPPGPIAEV* 355

DmTIPE NG--AISTSNSVQGNLSKTMTTSISTPPGPTAAV* 453

B
PaTipE
DmTipE
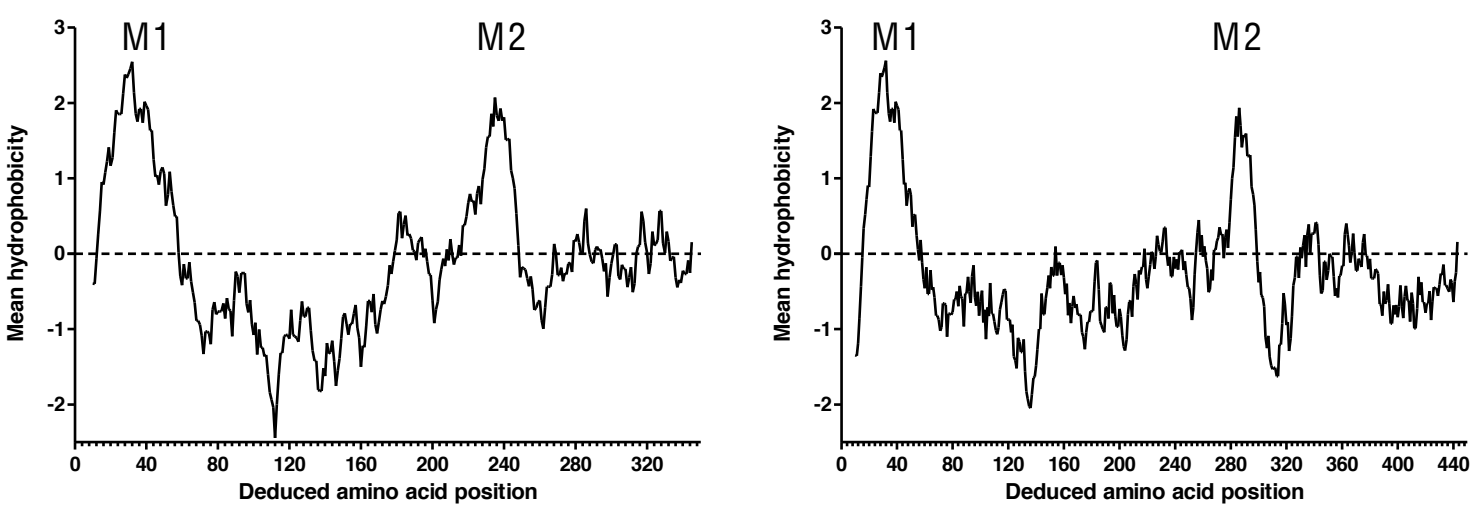

C

D

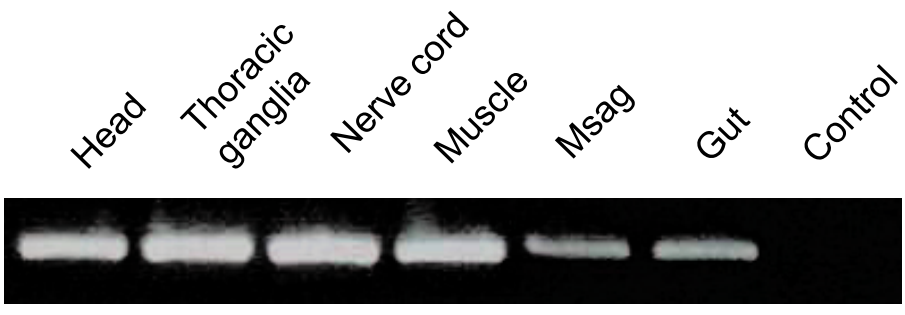

PaTipE

(1065 bp)
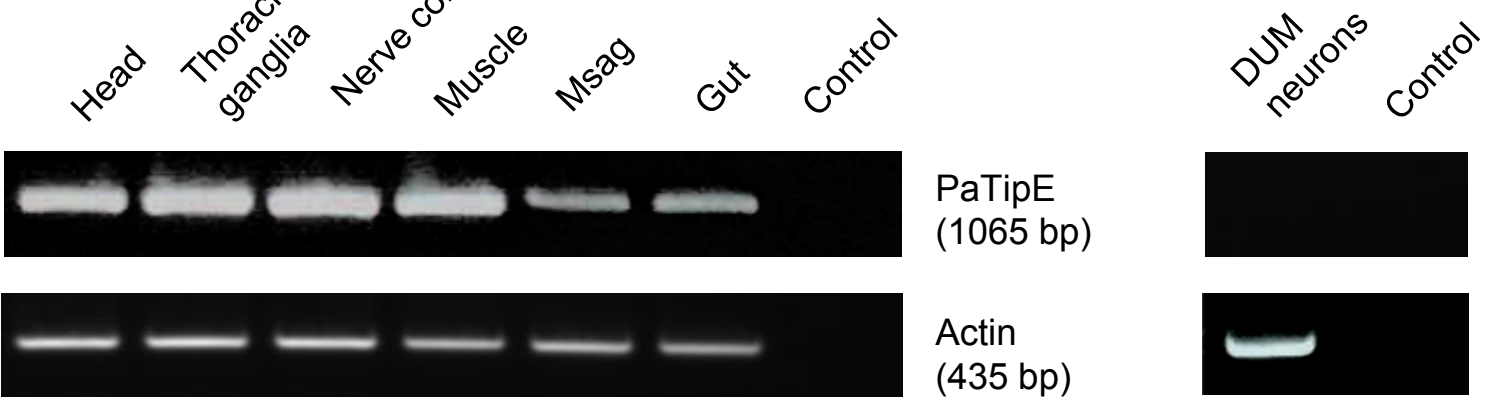

Actin (435 bp)

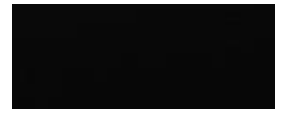

PaTipE (1065 bp)

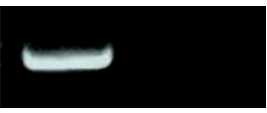

PaTEH1 (840 bp)

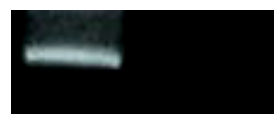


A

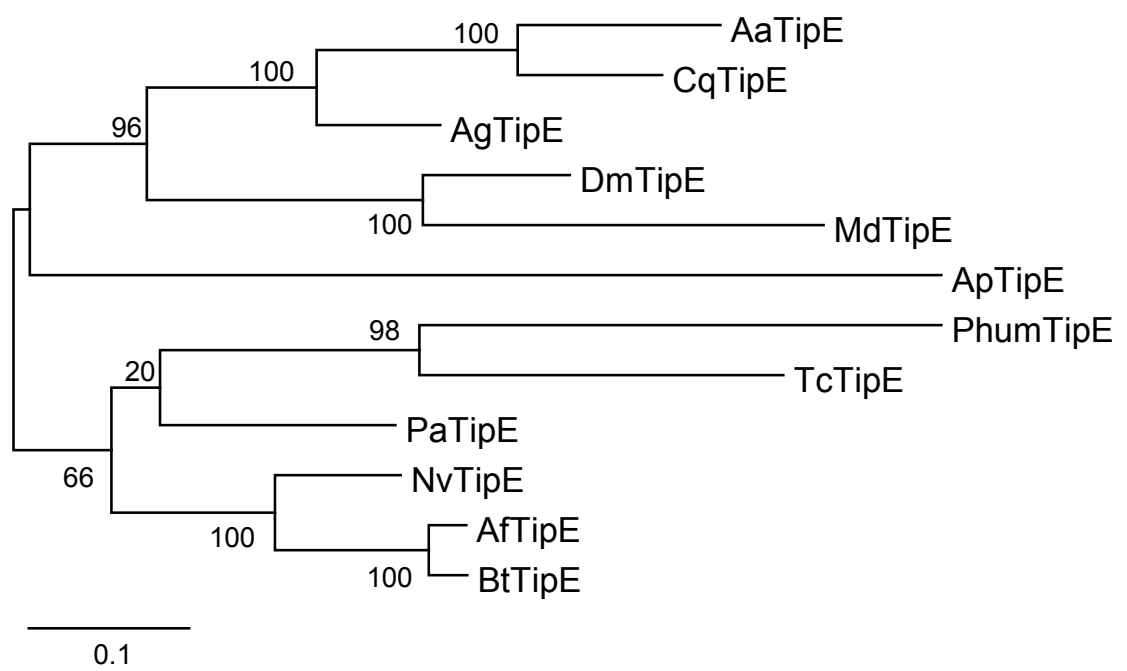

B

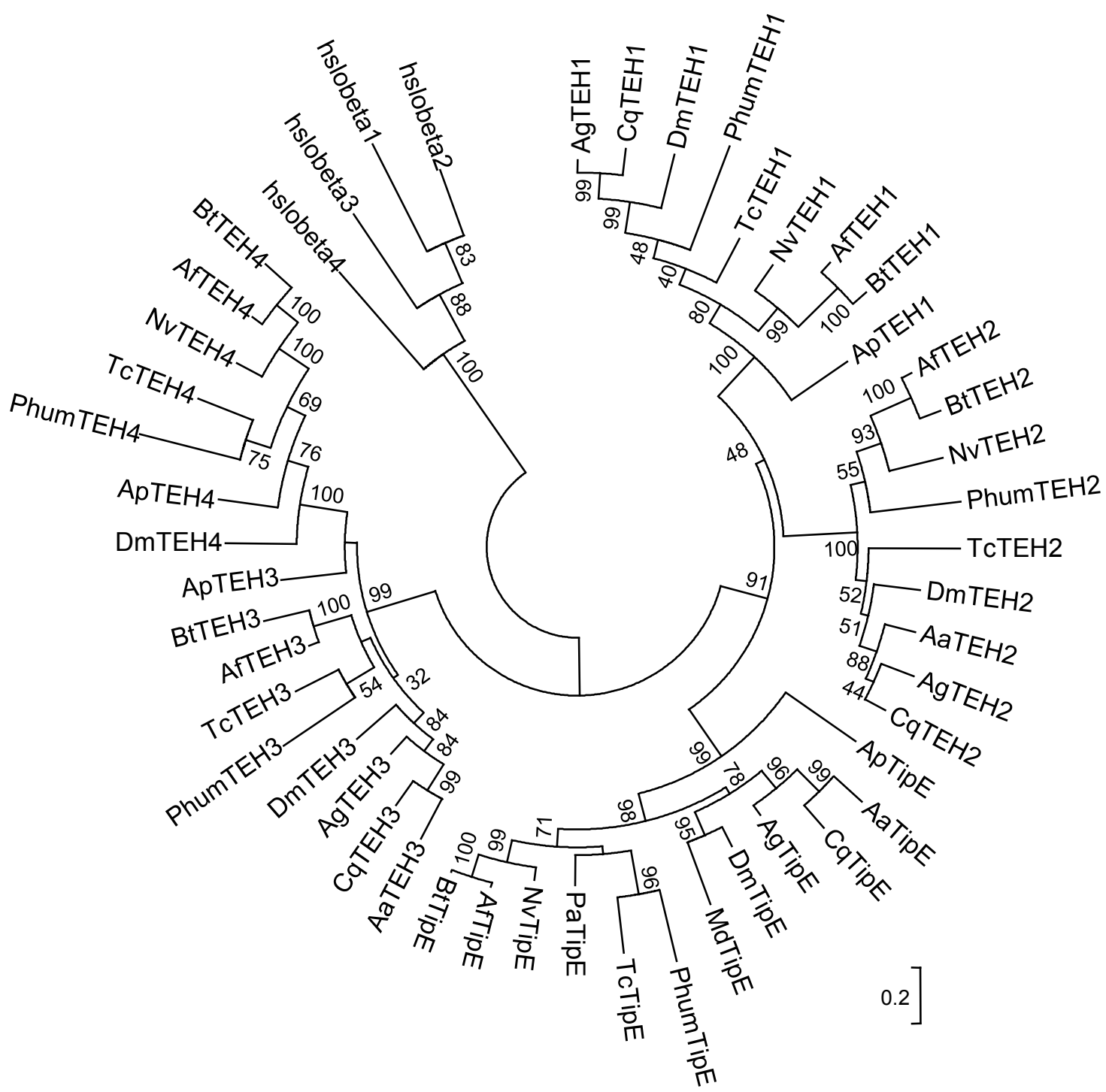


A

$\mathrm{PaNa}_{\mathrm{v}} 1$

$\mathrm{PaNa}_{\mathrm{v}} 1+\mathrm{DmTipE}$

$\mathrm{PaNa}_{\mathrm{v}} 1+\mathrm{PaTipE}$

$\mathrm{DmNa}_{\mathrm{v}}$ 1-1

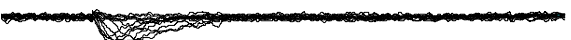

$\mathrm{DmNa}_{\mathrm{v}} 1-1+\mathrm{DmTipE}$

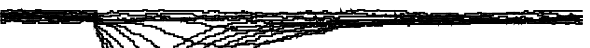

$\mathrm{DmNa}_{\mathrm{v}} 1-1+$ PaTipE

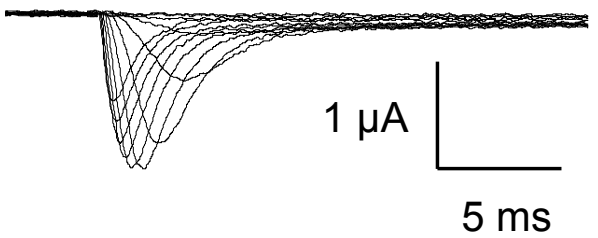

B

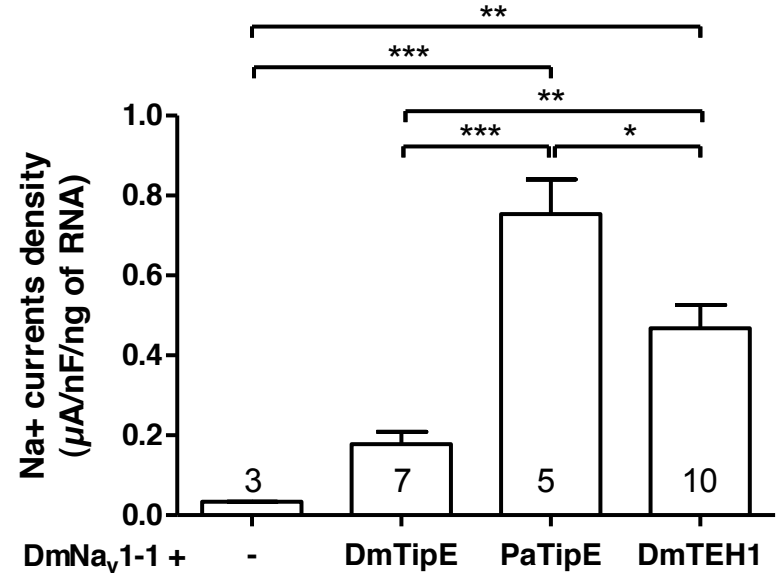


Figure 4

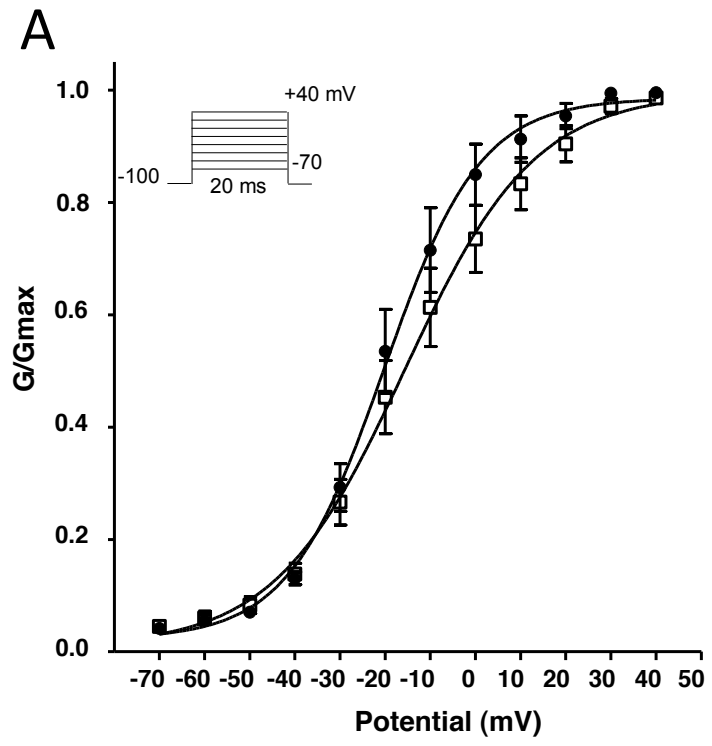

C

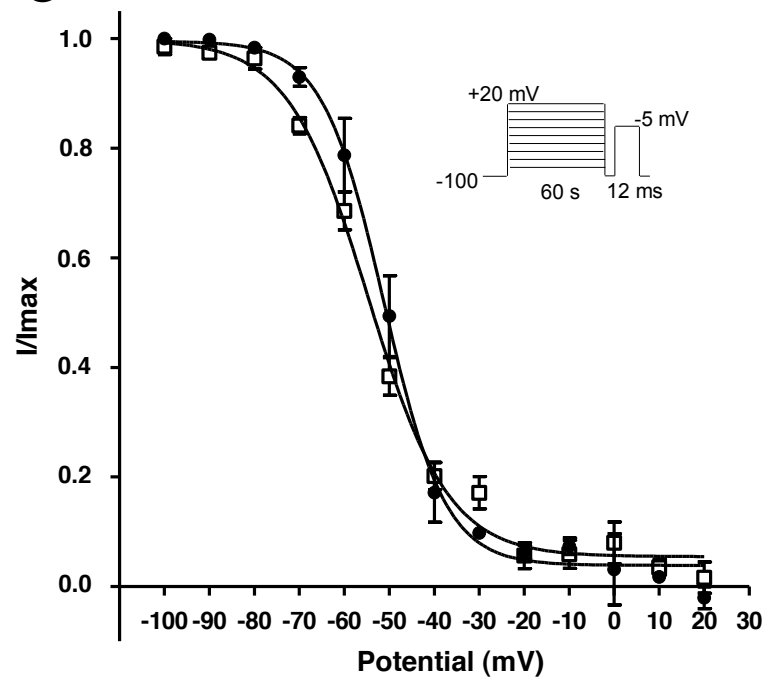

B
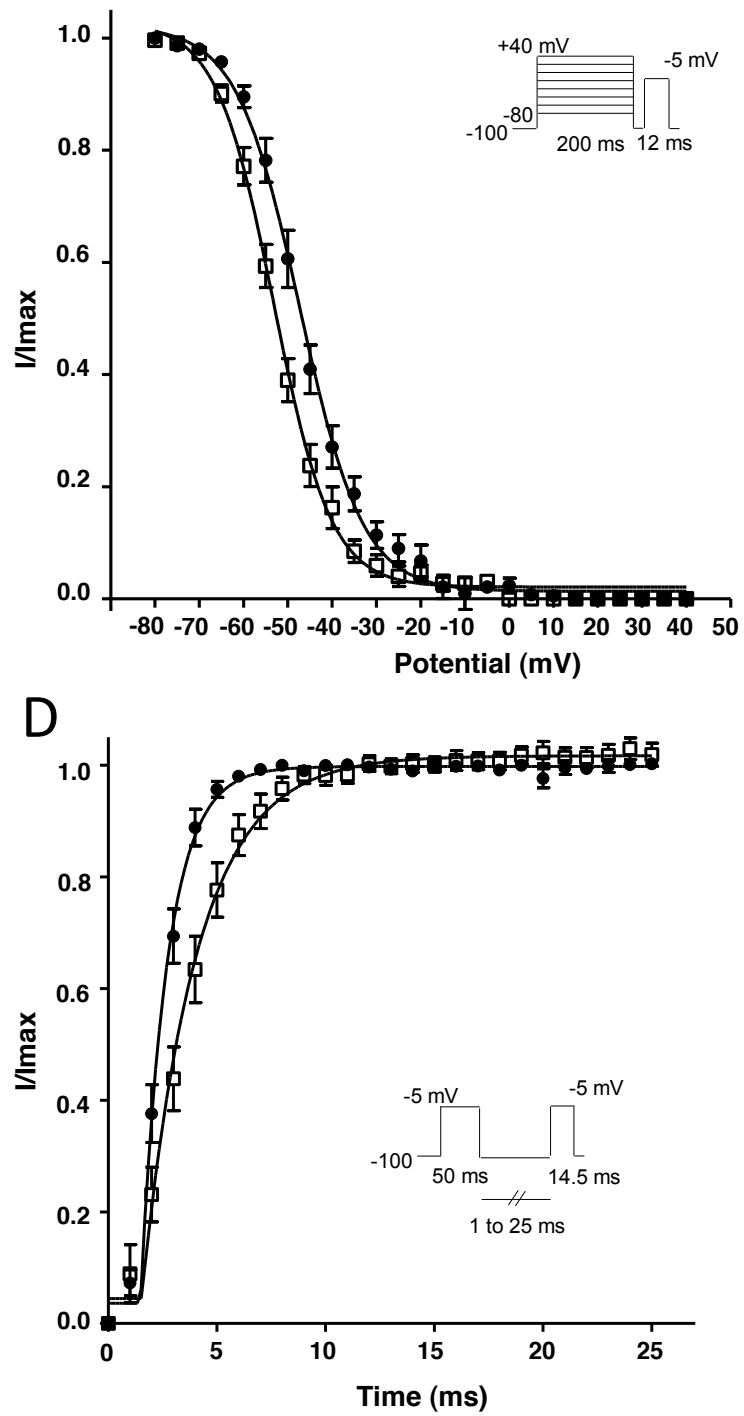
Table S1

Accession numbers of sodium channel auxiliary subunits used for the phylogenetic analysis

\begin{tabular}{|c|c|c|c|c|c|c|}
\hline Specie Names & Prefix & TipE & TEH1 & TEH2 & ТЕH3 & TEH4 \\
\hline Acyrthosiphon pisum & $\mathrm{Ap}$ & XM_003247842 & XM_001944580 & - & XM_003247843 & XM_003247846 \\
\hline Aedes aegypti & $\mathrm{Aa}$ & XM_001654675 & - & XM_001654677 & XM_001654676 & - \\
\hline Anopheles gambiae & $\mathrm{Ag}$ & XM_563317 & XM_310354 & XM_312094 & XM_312093 & - \\
\hline Apis florea & Af & XM_003697956 & XM_003689858 & XM_003697957 & XM_003697954 & XM_003697996 \\
\hline Bombus terrestris & $\mathrm{Bt}$ & XM_003393369 & XM_003393464 & XM_003393371 & XM_003393372 & XM_003393368 \\
\hline Culex quinquefasciatus pipiens & $\mathrm{Cq}$ & XM_001845887 & XM_001847934 & XM_001845889 & XM_001845888 & - \\
\hline Musca domestica & $\mathrm{Md}$ & AF131734 & - & - & - & - \\
\hline Nasonia vitripennis & $\mathrm{Nv}$ & XM_003425721 & XM_003425719 & XM_001606821 & - & XM_001606830 \\
\hline Periplaneta americana & $\mathrm{Pa}$ & KC992733 & - & - & - & - \\
\hline Pediculus humanus corporis & Phum & XM_002430448 & XM_002430447 & XM_002430450 & XM_002430449 & XM_002430451 \\
\hline Tribolium castaneum & Tc & XM_964916 & XM_964728 & XM_964775 & XM_964845 & XM_964710 \\
\hline
\end{tabular}


gaacttctttagttacaagccggggcatgggtaggtttggcaacgcagagtggaggcgggagattttagagcgatattgtgtttgctca 90

ttgctttcgttatacgtaacgcgtatttttcaatattacttcatgcacaaagggtgcattcaaagcacgatgacttatagatggaggta 180

gggtgcgatgcgggcggtctaacgcgcgtgcgcCATGGATGAGCCGGAGATGAGCGGACTTTGGTGCAGAACTGTTGTTCTACACCAC 270 $\begin{array}{lllllllllllllllllllllll}M & D & E & P & E & I & E & R & T & L & V & Q & K & L & L & F & Y & T & T\end{array}$

СGCTTTCTTCGTCCTCCTTGGCACTTTCGGCCTCTTCGCCTTCCTCTTCCTGGTGCCTTTCGTCATCGATCCGGCCTTCACGACCATCTT 360 $\begin{array}{lllllllllllllllllllllllllllllllllll}\text { A } & F & F & V & L & L & G & T & F & G & L & F & A & F & L & F & L & V & P & F & V & I & D & P & A & F & T & T & I & F\end{array}$ CATGCAGTTCGACCCTGTGGCCGCCCTTTGCCAGACGGTGCATACCGAAACGAGGCATGGCGCGTCCAATTGCACGTGGAGCTCCTGTAG 450 $\begin{array}{lllllllllllllllllllllllllllllll}M & Q & F & D & P & V & A & A & L & C & Q & T & V & H & T & E & T & R & H & G & A & S & N & C & T & W & S & S & C & R\end{array}$

AGAGGGCTGCACCAAGGAGGTGTACGAGTGCACGCAGATTCGGGTCAACTACAAAATTCTGCCGCCAGTTAAGCAGGAGGAAGGCGAGGA 540

$\begin{array}{lllllllllllllllllllllllllllllll}E & G & C & T & K & E & V & Y & E & C & T & Q & I & R & V & N & Y & K & I & L & P & P & V & K & Q & E & E & G & E & E\end{array}$

GGGGAATGCTGGGGAGGACGAGGGGACGGATGAGGACATCGTGGCGGAGCTCCACGAATATCTGCGGTCCAAGAGAGCTATCCGCGACTA 630 $\begin{array}{llllllllllllllllllllllllllllllll}G & N & A & G & E & D & E & G & T & D & E & D & I & V & A & E & L & H & E & Y & L & R & S & K & R & A & I & R & D & Y\end{array}$ CGACTACTCGAGCGAGGACGAGGAGATCGCCCTGCAGGAGCCGGAGCCCACGGGACTCATGGGGAACGACTCGGAGTGGTATTATACCCA 720 $\begin{array}{llllllllllllllllllllllllllllllllll}D & Y & S & S & E & D & E & E & I & A & L & Q & E & P & E & P & T & G & L & M & G & N & D & S & E & W & Y & Y & T & Q\end{array}$ GGCGAAGCTGTTCCCCAACGTGAAGGGTTGCGGCTACCCGCCCATGCTCAACTGCAGCATCTTCGTCAAGATGTACAGTGAACCGGGGAC 810

$\begin{array}{lllllllllllllllllllllllllllllllll}A & K & L & F & P & N & V & K & G & C & G & Y & P & P & M & L & N & C & S & I & F & V & K & M & Y & S & E & P & G & T\end{array}$

CAACTTCTCGTGCTACTACAGCAAGGTGGATCCGGGGCTGGTGATGAGCGAGCTGGACATGTGGCAAGTGTATATGAATCTCGTATACGC 900

$\begin{array}{lllllllllllllllllllllllllllllllll}N & F & S & C & Y & Y & S & K & V & D & P & G & L & V & M & S & E & L & D & M & W & Q & V & Y & M & N & L & V & Y & A\end{array}$

GATGGCCATCCCCATCCCGTCCTTCATCATCTCCGTCATCTACCTGACCTTCGCCTACTTCAAGATCTACAACGACGACGACGAGGAGGC 990 $\begin{array}{lllllllllllllllllllllllllllllllllllll}M & A & I & P & I & P & S & F & I & I & S & V & I & Y & L & T & F & A & Y & F & K & I & Y & N & D & D & D & E & E & A\end{array}$ CCTCGTGAATGCGGCCGGGGAGACGGTGGAGGGAGACGCCGCCGAGAACGGCGTGACGCCGGTGCCGCCTCTCACGAGCGGCGCCATCAC 1080 $\begin{array}{llllllllllllllllllllllllllllllllllll}L & V & N & A & A & G & E & T & V & E & G & D & A & A & E & N & G & V & T & P & V & P & P & L & T & S & G & A & I & T\end{array}$ GCCGGGCAGCGAGATCTTCAGGGAGGATCTCGCGAGCTTCGGGCACCAGCTCAAAGTGGCGATGGCAGACGAGATGAGCAGAGAGAGTGT 1170 $\begin{array}{llllllllllllllllllllllllllllllll}P & G & S & E & I & F & R & E & D & L & A & S & F & G & H & Q & L & K & V & A & M & A & D & E & M & S & R & E & S & V\end{array}$ AGCGGATGCCGTCCCCGTCATCGCCAACTCAAACTCCATACCTGGAAACTTGAGCAAGACGATGACGACCAGTATCTCAACGCCGCCTGG 1260 $\begin{array}{llllllllllllllllllllllllllllllllll}A & D & A & V & P & V & I & A & N & S & N & S & I & P & G & N & L & S & K & T & M & T & T & S & I & S & T & P & P & G\end{array}$ GCCGATAGCGGAAGTCTGAagaagtccttccatggcctgtccaacatctcctggagacccaagtaaccagctgtgagtctcggatcgggc 1350 $P \quad I \quad A \quad E \quad V \quad$ *

aatttgaaacataacatagcaataccaggaacttgtaggtgtacataatcgcttgatttcattcactatcggatgtcaacatttgtgttt 1440 tqctttttattqcacatatqtacaqqaqaqaqaatqtqaqaqcttqtqtatqcaqaaqaattaqccacttqaatttqttcaaqaatqa ta 1530 tatacatatatatatttataatacgcaatattaataccctaactgtacttgcaaacacaccacactctgaaatatctgccacaggctt 1620 taaccttqtqtqqcattqcqtttqttttcttcaacttatatttttcaqqaaactttqaaqtaaactttactctctacactaaqttqttta 1710 ttgttagggaatttgaataaatctgaagaccataagaaataagtattttgtaagtttcgaaattcgtgtggcttaataatgactctatct 1800 tgtttaaattttgaaaacaatataatcaagaatggaattttcatcttctgaagtattgtatgataacaaatataaagcttaacggct 1890 tgtattgcagaaagacaattgagctctttattttcgacgctcttgtcactgttttaaagaatatgaacttaatttcatacagagacacg 1980 


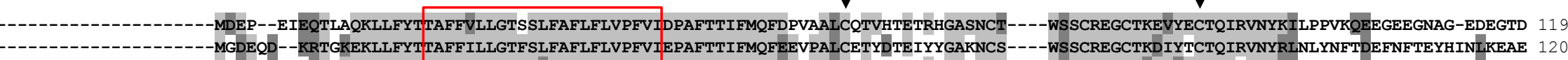

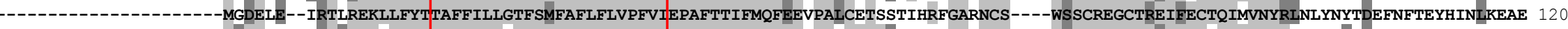

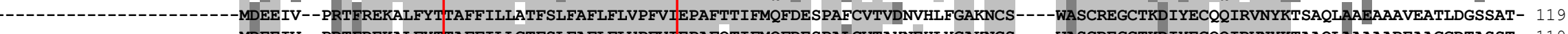

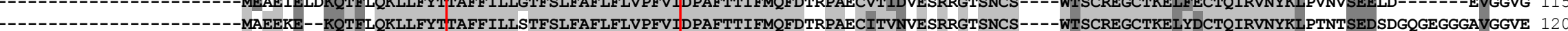
-------------------MAEEKE--KQTELQKLLFYTIAFFILISTFSLFAFLFLVPFVIDPAFTTIFMQFDTRPAECVTIDVESRRGTSNCS----WTSCREGCTKELYDCTQIRVNYKLPTNTSEDSDGQGEGGGAVGGVE 120 MDDDGPAAVDDAAAAIEKQLEQQQREEAERKRLEELRERVLFYI TTFFILLGIVSLFVFLFLVPFLIEPAITTLLMEFDETPTTCVTAYSQIREGASNCSLPGGWASCREGCTREIYECAQIFVNYTVPEDRAGDLNARHRRSLIVRGYR 150

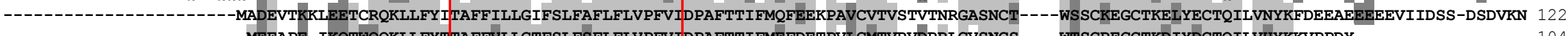
---------------------MFEAPE-IKQTWQQKLLFYTTAFFVLLGTFSLFSFLFLVPFVIDPAFTTIFMEFDETPVLCMTVRVDRRLGVSNCS----WTSCREGCTKDIYDCTQILVHYKKVPPDY------------------ 104

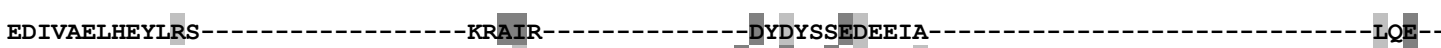

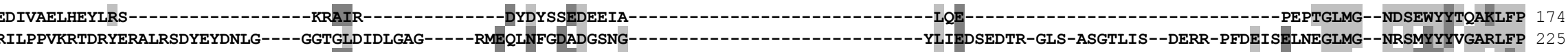
KVIPPKKRTDRYTRAIR-DYEYD-LGPHDPMAAAMDDEGGGGGGGGMVMEHLNFGDEDGSNG------------------------FLIEDS-----GLDPDSDTLIN--ADGSDVFEEISELNRGLMSS-NDSKYFFVGAHLYP 231 STVASKAATATATKTSGDSSVNN-----DNSRSSLKNTLDSLSGNSETNKPISITDRDSSSS-----------------------------LVNG-------FGDHKNGLISSIIGEDNTLMEYPDDMTEMNG--NESEWEFTGARLFP 225 PAPSTAAGTSASTNPTGDSSISREESSRDNSIASSSNSVAKPGGGGSSSOPISTSDGGGGASRALAGSNSRYGSALNRFERAIRADYDYGDALLEGGNGLGGGYGDSKHGFISSIIDDENAFIEYPEEMTGLMG--NNSEWYFTGARLYP 267 STVASKAATATATKTTGDSSVSN-----DSSKSSLTNNLNSDSDNSKTNKPISTADQESSAG--------------------------LYDSSK----NTGSCHRTAYSSLWRR-YTLIEYPDDMTELNG--NESEWFFTGARLFP 228

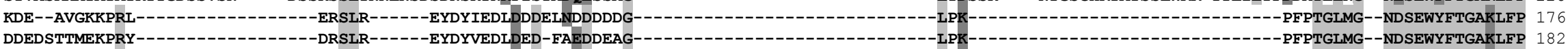
DDEDST

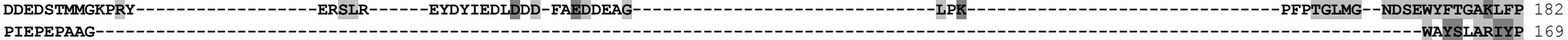
PIEPEPAAG----

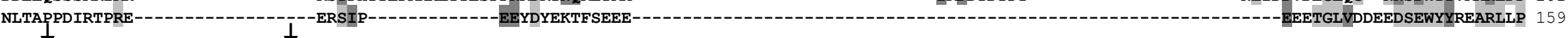

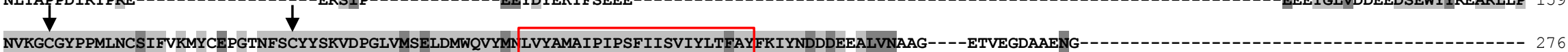
NVKGCGYPPMLNCTIWLKRYTKIGMKFPCYYSKVDPSLVISDLDYWQNTLNLVYSMAIPIPSFIISVIYLTYAYFKIYNEDEETAPLDKNAEDMDIDDIDAVDDSDGAVLADNVAGSOI INM-DSTTNDS--CLEG-------------- 358 NVKGCGYPPMLNCTIWLKRYTKIGMKFPCYYSKVDPSLVISDLDYWQNTLN LVYSMAIPIPSFIISVIYLTYAYFKIYNEDEETAPLDKNAEDMDIDDIDAVDDSDGAVLADNVAGSQIINM-DSTINDS--CLEG-NVKGCGYPPVLNCTIWFKRYTKLGVKFPCYYSKVDPSVVISELDYWQNTLNLIYSMAIPIPSFIISVIYLTYYAYFKIYNEDEETAPLDKNAEDMDIDD-EGVDDSDGAALADNAAGSQVINMHDSTTGES--CIVDG-------------- 365 NVKGCGYPPILNCTIWTKKYWKIGTNFTCFYSRVDPGLVISDLDMWQNTLN LVYAMAIPIPSFIISVIYLAFAYFVIFNEDEEAALIDKNGEEEEGIEDEMDETDEGNDNKIN-ESEAEITANHISDNN---VVEDG-------------H 359 NVKGCGYPPILNCTIWTKKYWTIGTNFSCYYSRVDPELVISDLDMWQNTLN LVYAMAIPIPSFIISVIYLAFAYFVIFNEDEEAGLLDKNGEDEAGEDDLEQETDEGNDNKINNENEVEVTANHINEKQQQQSADEENGVHTTTTTTPIQ 417 NVKGCGYPPMLNCTIWTKKYWKIGNNFTCYYSRVDPGLVISDLDMWQNTLN IVEAMAIPIPSFIISVIYLAFAYFVIYNEDEEAALLDKNGEEE-GTEDELAETDEGNDNKIN-ESEAEITANHINENN---VDGGGNTAPTTTTTPVQQ 373

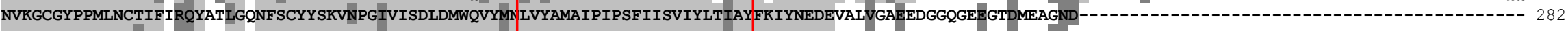
NVKGCGYPPMLNCSIFYRQYAIIGQNFSCYYSKVDPGIVISDLDMWQVYMN LVYAMAIPIPSFIISVIYLTIAYFKIYNEDEVVLVGGEE----GEEGGEGEGSN-------------------------------------------- 283 NVKGCGYPPMLNCSIFYRQYANIGQNFSCYYSKVDPGIVISELDMWQVYMN LVYAMAIPIPSFIISVIYLTIAYFKIYNEEEVVLVGGE----GEDGGEGEGSN------------------------------------------ 283 NVKGCGYPPHLNCTEFRNRYFEVGASYPCYYSRKEPWVVITELDLAKSTROLVYSMVFPIPCFVVVVYVALAYFCVYAGRHRKPKVKRRVVRRMGGGGATGSGAGYGPGGSASRAKSAGSEATPLTNNSGAAAAPSSPTGTG-TPGSE 318

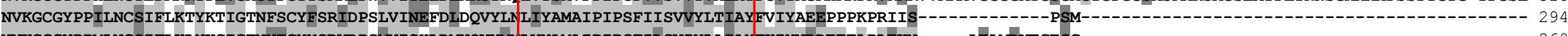

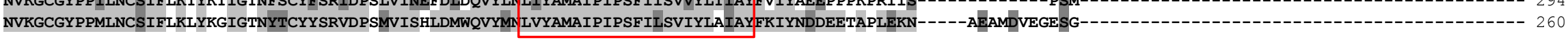

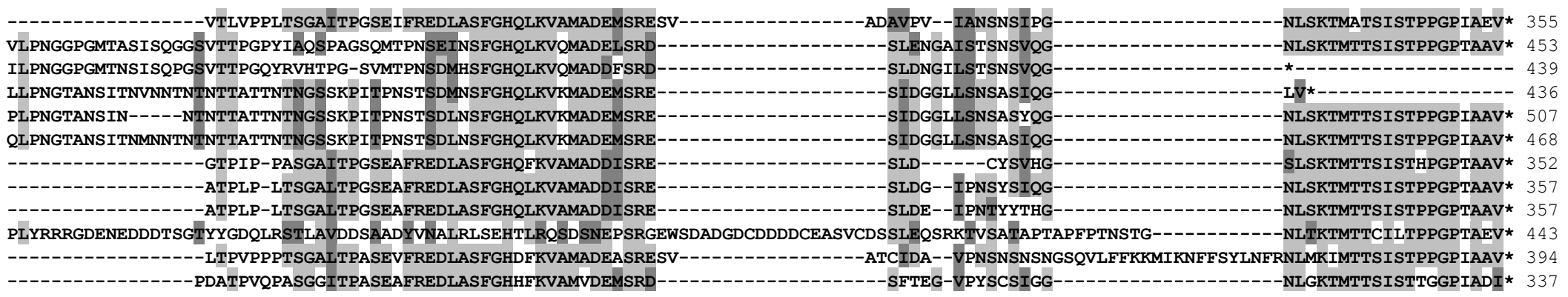



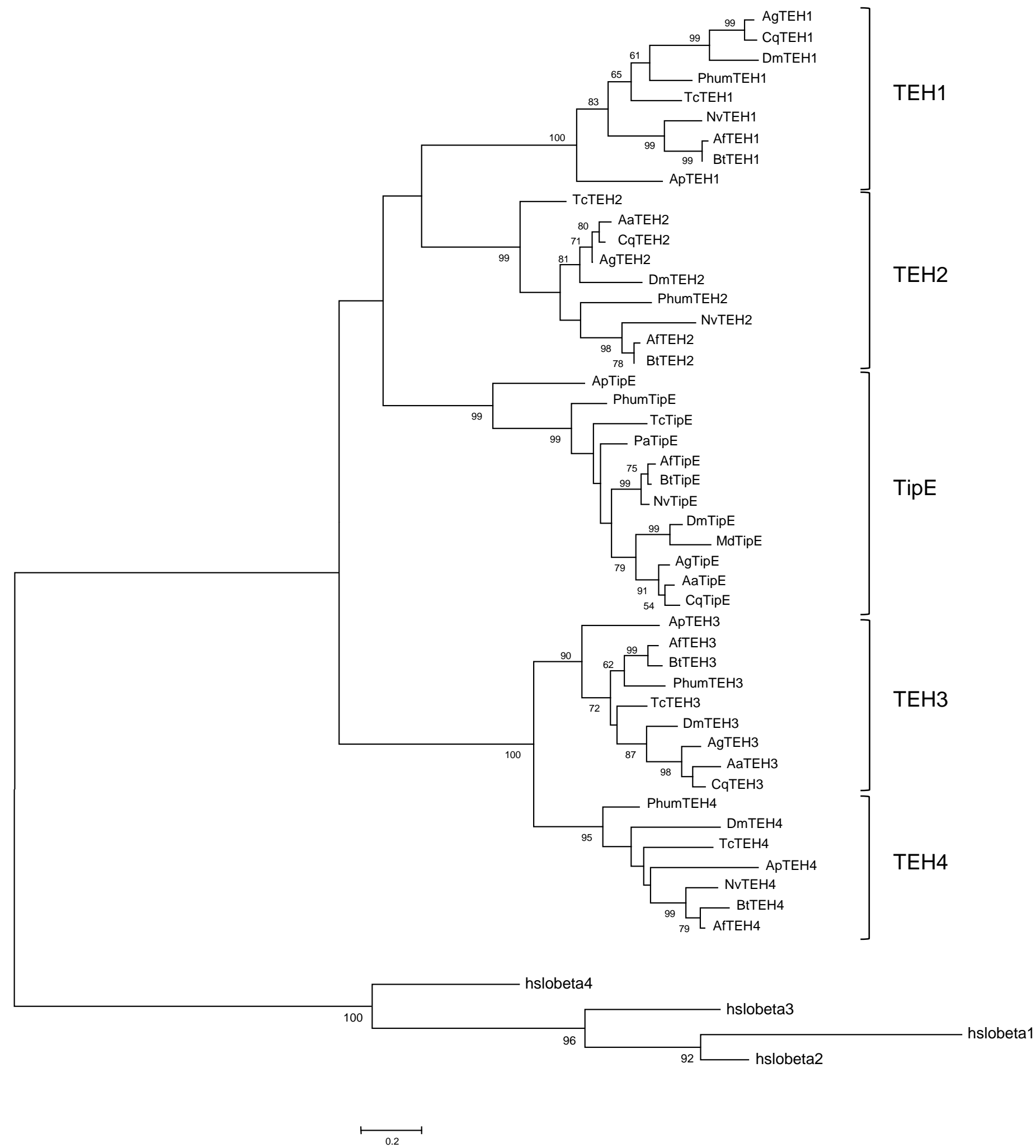

Figure S3 